\title{
P-1085 - Experiences of adolescents \& caregivers about structured education program 'Kids In Control OF Food (KICk-OFF) in Kuwait
}

\author{
Authors: N. Taha ${ }^{1}$, Z. Rahme ${ }^{1}$, N. Mesbah ${ }^{1}$, F. Sukkar ${ }^{1}$ \& A. Shaltout ${ }^{1}$ \\ Affiliations: ${ }^{1}$ Dasman Diabetes Institute, Kuwait.
}

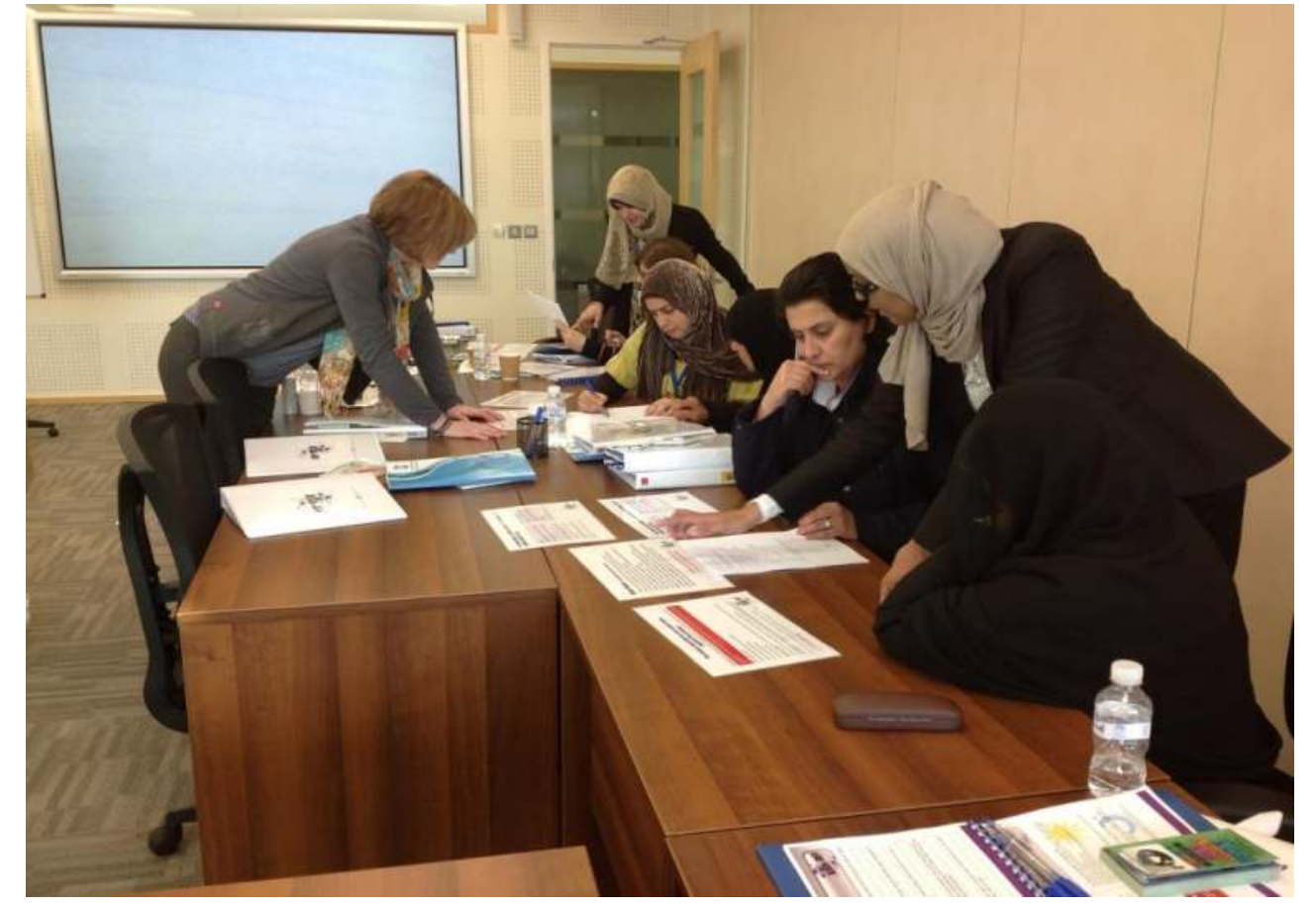

Background

Kids In Control OF Food (KICk-OFF) is the paediatric version of 'Dose Adjustment For Normal Eating Programme (DAFNE)". It is a diabetes Structured Education Programme (SEP) that is provided over five full days for young people with type 1 diabetes, to empower them with the key diabetes self-management skills that they need to acquire and implement in their day-to-day life in order to get better glycemic control. To date it would appear that no qualitative studies have been published regarding the attendees of the KICk-OFF course neither in United Kingdom (UK), where the course originated, nor in Kuwait where it commenced in January 2013. Thus, it was necessary to investigate the impact of this imported learning structured education programme on glycemic control and self-care behaviors through patients' and caregivers' perspectives.

\section{Aim}

To explore the perceptions and experiences of adolescents and their caregivers towards 'Kids In Control OF Food (KICk-OFF)", a diabetes structured education program that is provided over five full days for adolescents (11-16 years old) with type 1 diabetes. KICK-OFF is designed to empower participants with diabetes self-management, knowledge and skills for better glycemic control.

\section{Method}

A qualitative exploratory case study. Semi-structured interviews were used to explore experiences of five adolescents and five caregivers. Participants were interviewed individually. Interviews were audiorecorded and then transcribed verbatim. Data was analysed using an inductive thematic approach.

\section{Results}

Five main themes emerged from the thematic analysis. 1-Value of the Program: interviewees reported that they acquired enough knowledge for better understanding of diabetes, their autonomy was enhanced, they are more efficacious and competent at diabetes self-management, and they can lead more flexible lives. 2-Confidence: adolescents and their caregivers gain more self-confidence, and hence they provide more support. KICK-OFF enhanced the sensation of control over the disease. 3-Psychological effect: KICK-OFF helped participants to better cope with diabetes. It helped reducing diabetes-related family stress and gave them more social freedom. It also supported behavioral change for better lifestyle. 4-Course setting and layout: all participants enjoyed the KICK-OFF guiding style. They all had positive overall impression about the course and suggested reinforcement courses 5Clinical Parameters: attending KICK-OFF led to better glycemic control and helped reduced the rate of hospital admissions.
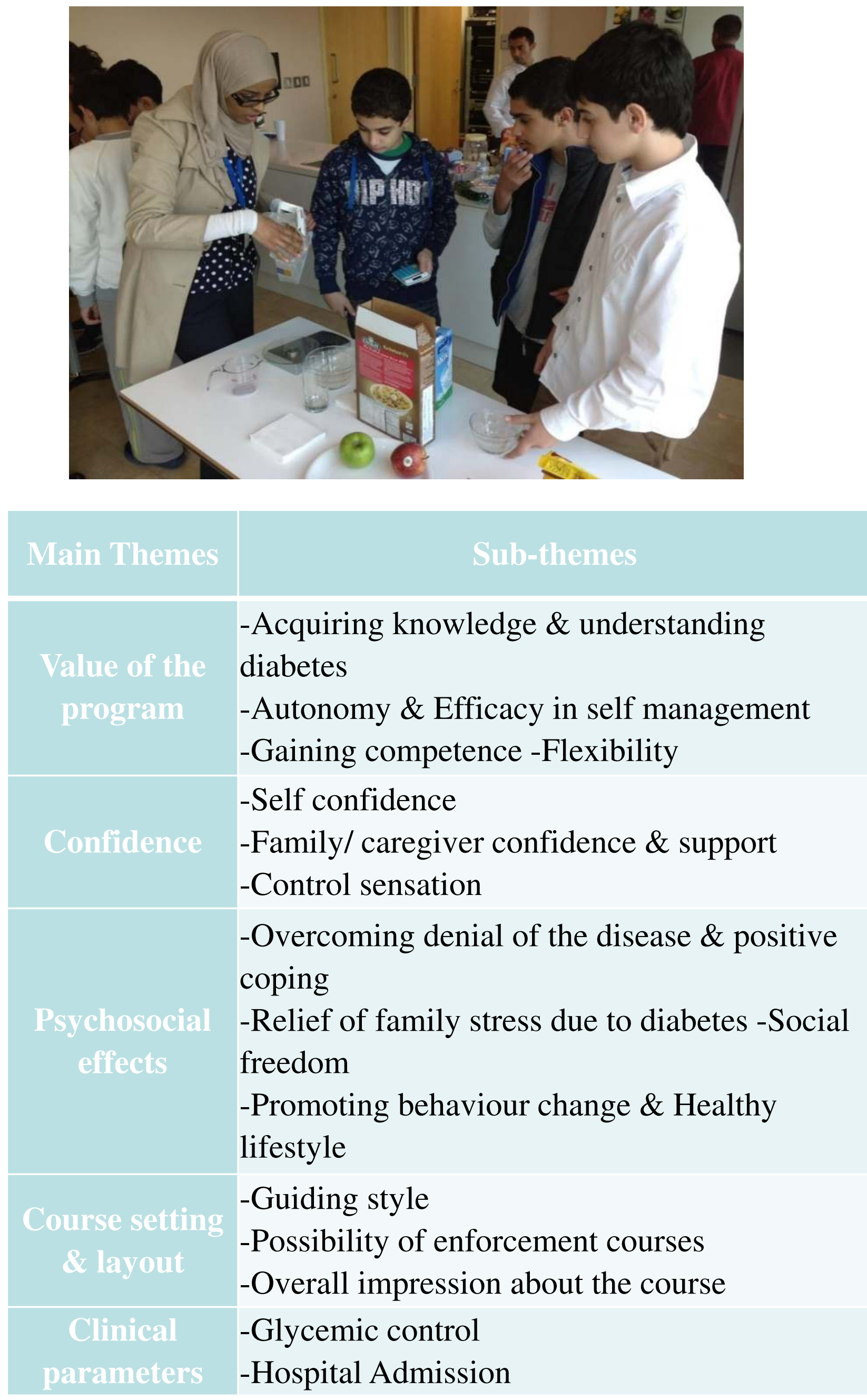

\section{Discussion}

Experiences of participants with type 1 diabetes and their Caregivers of KICk-OFF as an intensive structured education program are positive. It was perceived as a valuable program that enhanced their confidence and psychosocial status, and resulted in a better clinical outcome. Possible follow-up courses would be of benefit to the attendees.

\section{References}

1. Waller, H., et al., Pilot study of a novel educational programme for 1116 year olds with type 1 diabetes mellitus: the KICk-OFF course. Arch Dis Child, 2008. 93.

2. Price, K.J., et al., Does an intensive self-management structured education course improve outcomes for children and young people with type 1 diabetes? The Kids In Control OF Food (KICk-OFF) clusterrandomised controlled trial protocol. BMJ Open, 2013. 3(1).

3. Price, K., et al., Effectiveness of the Kids in Control of Food (KICkOFF) structured education course for 11-16 year olds with Type 1 diabetes. Diabetic Medicine, 2016. 33(2): p. 192-203.

\section{Acknowledgements}

Dr. Azza Shaltout, Dasman Diabetes Institute Dr. Katherine Price, Sheffield Children's Hospital Julie Knowels, Sheffield Children's Hospital 